\title{
Method for analysing the deformation and stress of vertebra assembly
}

\author{
Alina Rodica Spanu ${ }^{1, *}$ and Daniel Besnea ${ }^{1}$ \\ ${ }^{1}$ University Politehnica of Bucharest, Mechatronic and Precision Engineering Department, 313 \\ Splaiul Independentei, Sector 6, Bucharest, Romania
}

\begin{abstract}
The paper presents a method for testing the deformation and stress values of the vertebra assembly when variable forces are applied on the body surface. The working points have very well-known positions and they could be changed, so the compression force, torsional or bending couples could act. The system is actuated by an electrical stepper motor controlled with Arduino. The force and displacement sensors were used.
\end{abstract}

\section{Introduction}

The lumbar spine is one of the main sections of human body due to its important role of providing the capability of maintaining the vertical position during walking, working and some other activities. Generally speaking, it is a part of our body with static and dynamic influence as well as the anatomic role. A per cent of $60-85 \%$ of population is afflicted with spine pains and this becomes a phenomenon even for young people, $40-45$ years old for instance [1].

The main problems that could appear causing some troubles and pains are degeneration process due to aging, some loads exceeding the normal values during working and leisure time or accidental situation causing injuries.

Regarding the forces acting on spine during movement or stationary periods, we have to mention at first the gravity, which is proportional with body mass. Meantime, this mass value influences the inertia force developed during accidental fallings amplified by the acceleration. Supplementary forces could appear when we move some objects by humping them, so the load becomes mechanical couple depending on the distance between the spine axis and the object. Ergonomic aspects, such as chair position in front of the table as well as chair shape, are brought about some distress too especially when we are sitting a long period without pause for standing or walking. At last we should mention the sedentary life at home watching TV set or doing something else.

There are many studies that analyse the influence of all these factors on the spine deformation, so the conclusion is the lumbar section is one of the most affected. The lumbar spine is the section of spinal column between the thorax and the sacrum. It consists of five vertebrae named L1 to L5 with their posterior elements and articular facet joints, of intervertebral discs, ligaments and the surrounding muscles.

* Corresponding author: spanu alina@yahoo.com 
The lumbar vertebrae are quasi cylindrical with a lateral width of 40-50 $\mathrm{mm}$ and sagittal depth of $30-35 \mathrm{~mm}$. The height of a lumbar vertebra is about $25-30 \mathrm{~mm}$. The lumbar vertebrae are thicker anteriorly than posteriorly. The intervertebral discs separate the adjacent vertebrae. They are quasi cylindrical with a lateral width of 40-45 $\mathrm{mm}$ and sagittal depth of $35-40 \mathrm{~mm}$. The height of the lumbar disc is about $10 \mathrm{~mm}$. The cartilaginous endplates separate the nucleus and annulus from the vertebral bodies and they cover almost the entire surface of the adjacent vertebral bony endplates. The plates have a mean thickness of $0.6 \mathrm{~mm}[1]$.

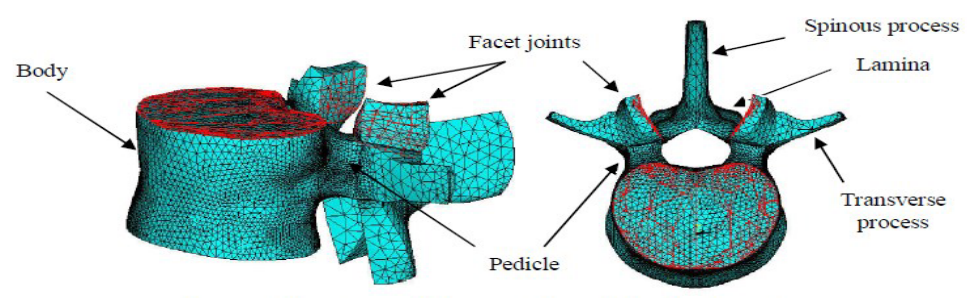

Fig. 1. Anatomy of the vertebra of the lumbar spine [2].

As we may infer from figure 1, there are the vertebra body, spinous process, transverse process, lamina and pedicle and facet joints. The layers of cortical and cancellous bone of the body are integrated in the same type of material only for computing the deformations.

The particularity of each person should be taking into account by various types of apparatus gathering biomechanical data by image processing and finding the vertebra dimensions using computation methods finally.

The study [2] presents Finite Element Analysis (FEA) of the three - dimensional model of L2 vertebrae, made by using SolidWorks environment that has been done with ANSYS 11.0 considering hyperextension loading. The material has linear and isotropic properties and tetrahedral solid element was used for the entire model. The applied force in axial direction was $460 \mathrm{~N}$ for a person of $80 \mathrm{Kg}$ and the level of stress was analysed.

The paper [3] presents the experimental study made on the vertebra under compression with variable force. There are two steel plates, up and below the vertebra and variable pneumatic force is acting by varying the supply air pressure. The force and occurring displacement are simultaneously monitored.

The goal of this paper is to analyse the deformations and stress level for the assembly of two vertebrae considering the values for mechanical couple by applying variable forces over the cylindrical body surface of the vertebra. We suppose there are the real working conditions during lumbar spine deformation, if the factors presented above are acting. We may analyse the working point positions on the body surface as the result of person movements. An experimental setup was done for pointing out the force variation and the positions of its applying points while the deformation was registered and analysed.

\section{Three dimensional model of the vertebra}

In order to analyse the stress and deformation whether the forces and couples are acting, it is necessary to make the 3D model of the vertebrae by using Catia V5R17 environment using the Generative Shape Environment due to the very complex spatial shape.

We aim to provide a realistic model as possible as we may asses it and the result is presented in figure 2 . 


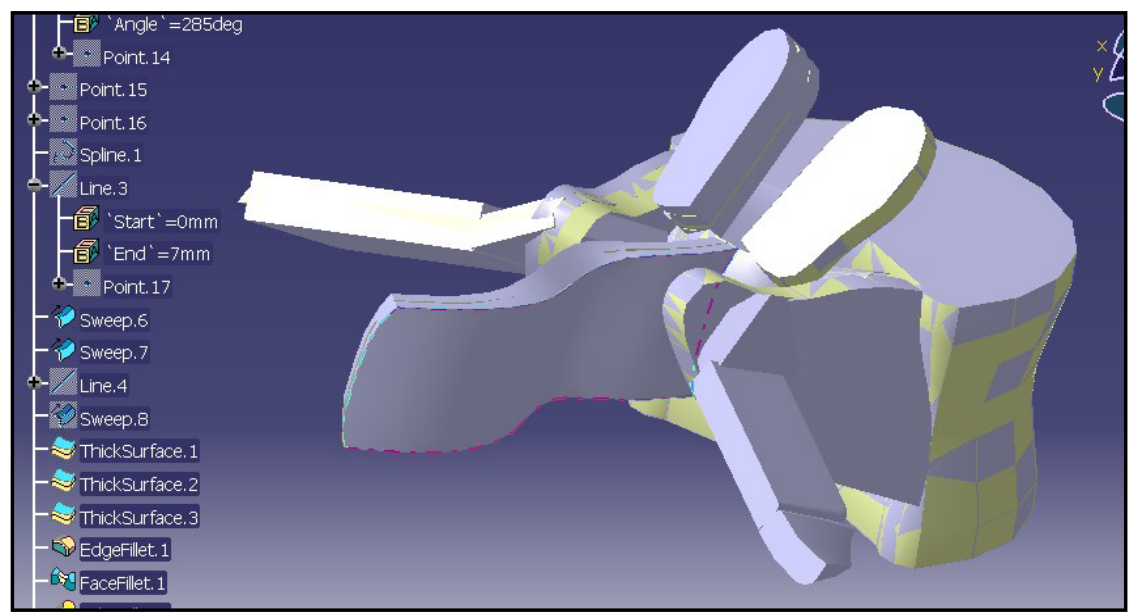

Fig. 2. The three dimensional model of the vertebra.

The next step was the rapid prototyping stage applied to the vertebra model by using the 3D printer - MakerBot Replicator. The main advantage of using the new technology of Additive Manufacturing is that it allows the user to manufacture the product directly from the $3 \mathrm{D}$ model in a very short period. Moreover, the spatial shapes could be very complex such them we aim to use for our experimental setup. The entire process is a cheaper one and the accuracy of the vertebra dimensions is good enough for later measuring.

After we have designed the model, it is transferred to the sectional processor and divided into a series of computed triangles. In figure 3 it is presented the manufactured model of the vertebra.

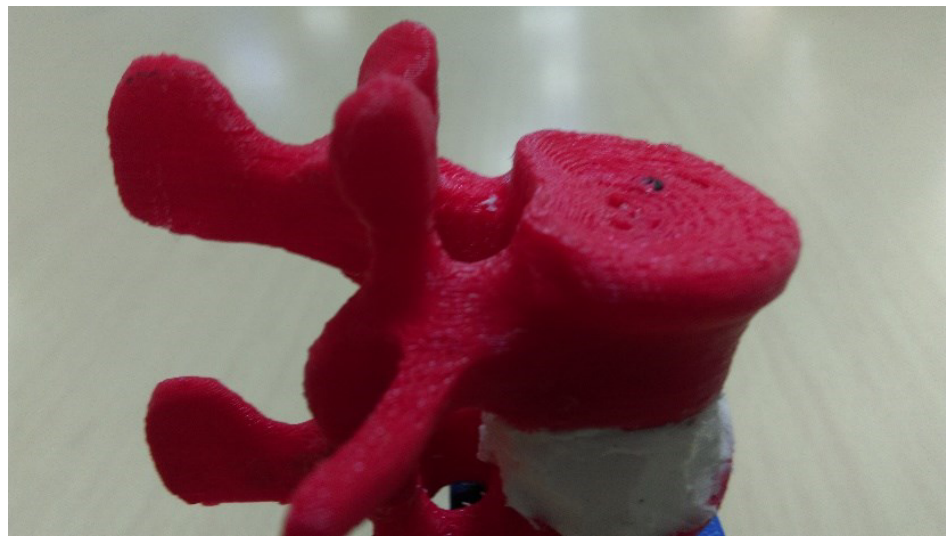

Fig. 3. The manufactured model of the vertebra.

In order to study the tensions and internal deformations during the forces are acting on the vertebra, we have design the profile of spatial cam - follower mechanism. The rotational cam is actuated by an electrical steper motor controlled with Arduino and the translational follower is developing the compression force acting on the vertebra.

The movement law is given by the equations below comprising the phase of starting the process, meaning the time $t$ is in the range of $t_{1} 0 \div 1.2 \mathrm{~s}$ and it is followed by the phase of ending the process, when the time is in the range of $t_{2} 1.2 \div 2.4 \mathrm{~s}$. Taking into account the way the maximum acceleration value influences the internal forces of this mechanism, we have imposed this value $y^{\prime \prime}(t)=0.8 \mathrm{~m} / \mathrm{s}$. The minimum value of acceleration has the main 
role in establishing the contact surface of spatial cam and follower, so that it may be computed with the equation:

$$
\mathrm{y}^{\prime \prime}{ }_{\min }=-\left(\mathrm{t}_{1} / \mathrm{t}_{2}\right) \cdot \mathrm{y}{ }^{\prime}{ }_{\max }
$$

The function of movement for the starting phase of spatial cam mechanism is given by the equation below [5]:

$$
\mathrm{y}=1 /\left(\mathrm{t}_{1} \cdot\left(3-\mathrm{t}_{1}-\mathrm{t}_{2}\right)\right) \cdot\left(3 \cdot \mathrm{x}^{2}-\mathrm{x}^{3} / \mathrm{t}_{1}\right)
$$

Meantime, we have computed the function for the last phase when the follower has to be stopped, as we may infer from the equation below:

$$
\mathrm{y}=1-\left(3 \cdot \mathrm{t}_{2} \cdot(1-\mathrm{x})^{2}-(1-\mathrm{x})^{3}\right) /\left(\mathrm{t}_{2}{ }^{2} \cdot\left(3-\mathrm{t}_{1}-\mathrm{t}_{2}\right)\right)
$$

The third dimension for describing the trajectory of the contact point between the cam and the follower is computed knowing the variation of angle $\Phi$, whose value is starting from zero degrees and its maximum value is $24^{0}$ in order to achieve the maximum and minimum value for ordinate y. It is expressing the level difference between the horizontal plane and the working plane.

Consequently, we have presented in figure 4 the variation of movement law $y(x)$ and in figure 5 the variation of cam profile depending on $\mathrm{x}$, $\mathrm{y}$ and $\Phi$.

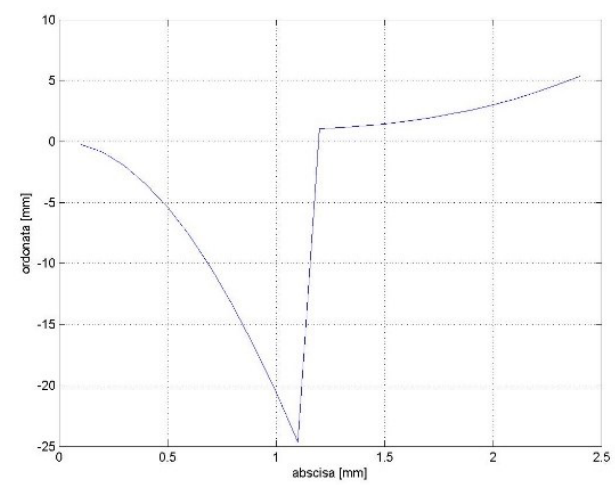

Fig. 4. The variation $\mathrm{y}$ as $\mathrm{x}$ function describing the movement law.

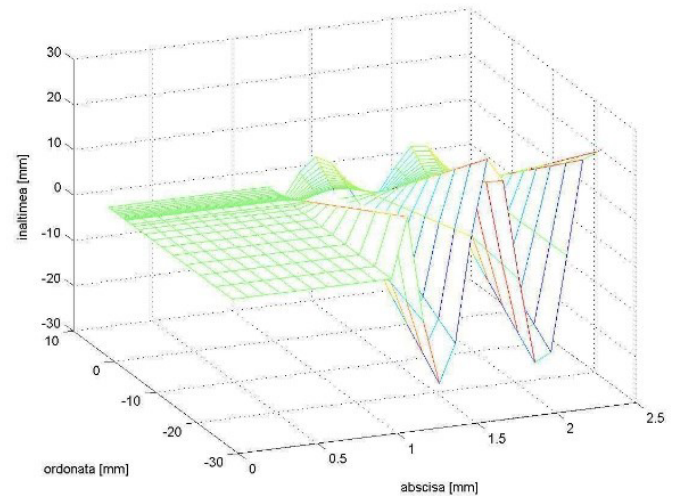

Fig. 5. The variation of cam profile. 


\section{Experimental set-ups}

Our goal is to analyse the stress and deformation values of the vertebra assembly 1 by measuring them, so that we have designed the system with three dimensional model presented in figure 6. It could provide a variable force applied in a well-known point position by using the electrical stepper motor 2 placed on a guiding subassembly 3 having an imposed accuracy.

The cam 4, actuated by the motor, provides the force acting on the vertebra body surface. Its working point position could be changed by motor displacement or by changing the vertebrae position, because of the particularity of placing it on the table 5 .

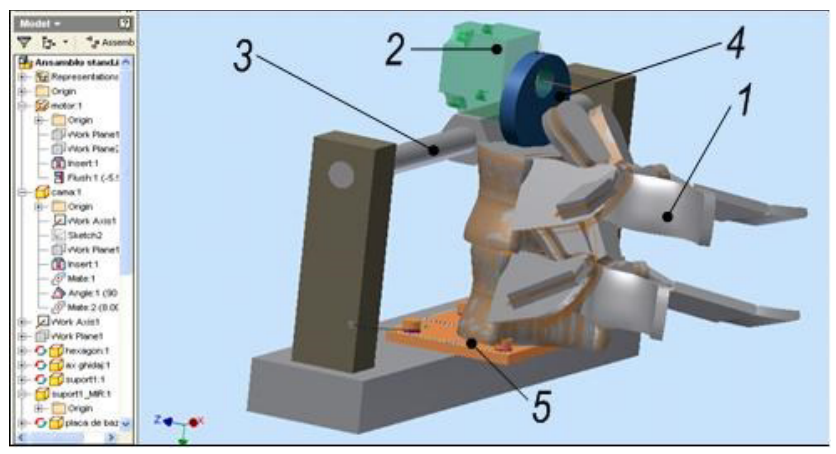

Fig. 6. The experimental setup of the system with variable force.

The main advantages of this solution are the variable and controlled force applied on the surface and the possibility of changing the working point position.

The most important force is the compressive normal one, but it is acting together with sagittal and lateral shear forces, so that the flexion/extension movement appears. Meantime, the bending and torsional mechanical couple values are generated. Consequently, the deformations of each element of the assembly appear and there are amplified by the disc with elastic properties between the two vertebrae.

The figure 7 presents the experimental set-up that we have made in order to measure the acting forces and the corresponding displacement of vertebra body. The system is actuated by the electrical stepper motor 1 , which could be moved along the $\mathrm{X}$ axis by the guiding system 2 .

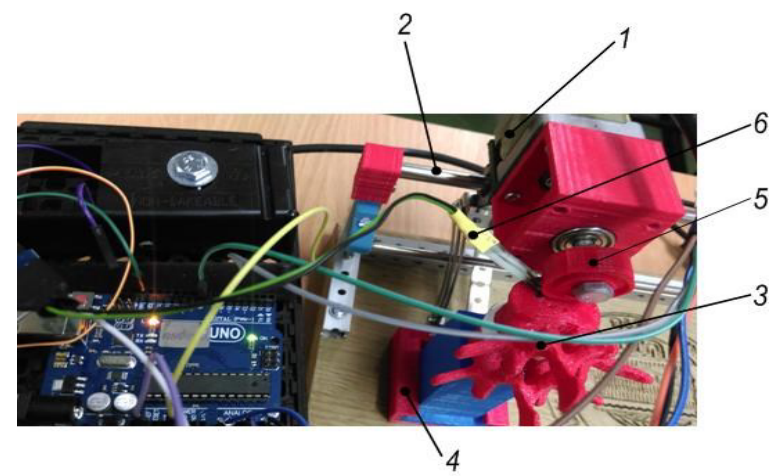

Fig. 7. The experimental set-up made for measuring the vertebra deformations.

The vertebra assembly 3 is put on the part 4 , so it could be rotated around $\mathrm{Z}$ axis. On the motor shaft is the cam 5 whose computed profile generates the variable forces applied on 
the points with very well-known coordinates. There are force and displacement sensors 6 for measuring the vertebra deformation, if flexion/extension movements are acting. We have used the FSR 400 force sensor, a very accurate one, with $4 \mathrm{~mm}$ its active diameter whose resistance is depending on how much pressure is being applied. We have used a 3 axis accelerometer/ 3 axis gyro with $\mathrm{I} 2 \mathrm{C}$ interface accesses for detecting the vertebra assembly deformation.

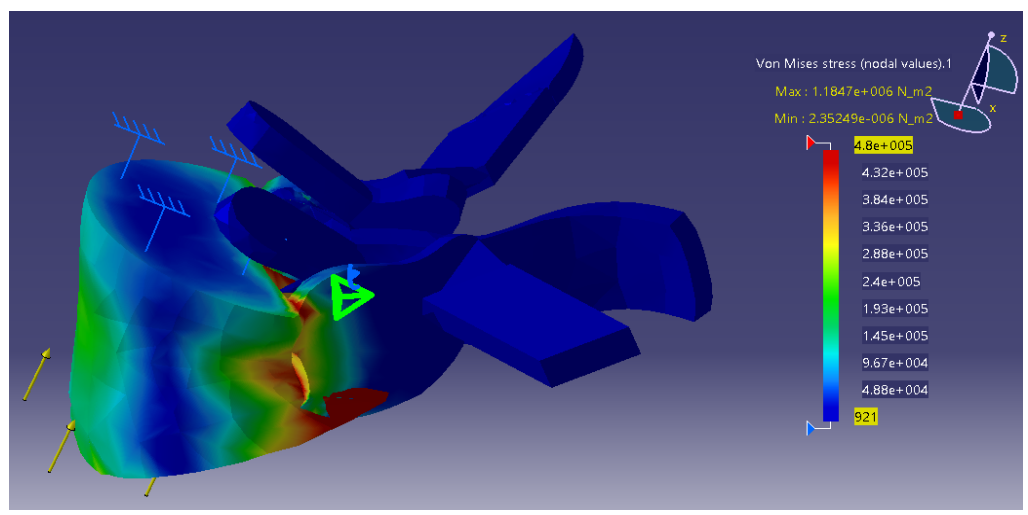

Fig. 8. The internal tension values according to Catia V5R17 Analysis.

In figure 8 we have shown the computed values of tensions applying the finite element analysis. In figure 9 we have presented the vertebra deformations when the compression force is acting.

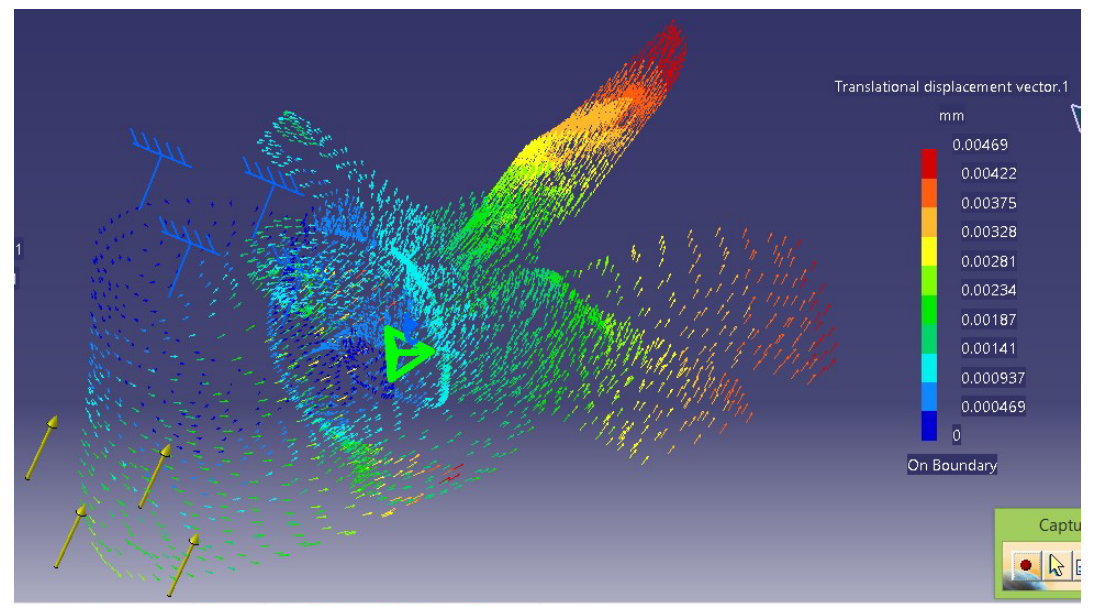

Fig. 9. The internal translational displacement of the vertebra.

\section{Conclusions}

The paper presents a method for deformation and stress measurement that could be applied for vertebra assembly. The main advantages of the proposed method are variable forces and mechanical couples provided by using a cam with computed shape actuated by controlled electrical stepper motor.

The three-dimensional model was designed according to the imposed demands and it was manufactured by FDM technology. We may determine the maximum values for deformation as force function. 
The future work will be focused on improving the experimental set-up solution considering compressing and torsional couple acting in the same time for a vertebra that has a material with properties ressembling with the human bone tissue.

\section{References}

1. M. Kurutz, L. Oroszvary, Finite Element Modeling and Simulation of Healthy and Degenerated Human Lumbar Spine, Finite Element Analysis - From Biomedical Applications to Industrial Developments, (ED D. Moratal, 2012).

2. A. Zulkifli, A. K. Ariffin, M. M. Rahman, IJAME 3, 256 (2011)

3. K. D. Bouzakis, S. Mitsi, N. Michailidis, I. Mirisidis, G. Mesomeris, G. Maliaris, A. Korlos, JMNJ, 4(2), 152 (2004)

4. J. Noailly, H. J. Wilke, J. A. Planell, D. Lacroix, J.B., 40(11), 2414 (2007).

5. I. Simionescu, V. Moise, A. Spânu, Mecanisme, (I.P.B., 1991) 